\title{
Canakinumab in the Treatment of Erosive Hand Osteoarthritis: A Case Series
}

\section{Carroll MB}

\author{
Keesler Medical Center, Keesler AFB, Mississippi, USA
}

*Corresponding author: Carroll MB, MD, FACP, FACR, 301 Fisher Avenue, Keesler AFB, MS, USA 39534, Fax: 228-376-0184, Tel: 228-376-3629, E-mail: mcar100210@yahoo.com

Citation: Carroll MB (2015) Canakinumab in the Treatment of Erosive Hand Osteoarthritis: A Case Series. J Case Rep Stud 3(5): 503. doi: 10.15744/2348-9820.3.503

Received Date: August 11, 2015 Accepted Date: October 10, 2015 Published Date: October 13, 2015

\begin{abstract}
Background: Erosive hand Osteoarthritis (EOA) is a common and debilitating form of hand OA for which there are no proven or efficacious therapies. Interleukin-1 beta (IL-1 $\beta$ ) plays a prominent role in OA joint inflammation. Canakinumab is a selective IL- $1 \beta$ inhibitor. The author hypothesized that canakinumab would be effective in alleviating the symptoms and functional limitations of EOA.

Methods: This prospective case series enrolled 3 patients with EOA who were treated with canakinumab 160 mg subcutaneously once. They were followed over a 12 week period, with visits at week 4 , week 8 , and week 12. It was anticipated that the effect of canakinumab would wear off and pain/function would return to baseline at week 12. The primary outcome of the study was improvement in QuickDASH at week 8. Secondary outcomes included changes in Health Assessment-Disability Index, subject reported pain, grip strength, and shirt button time. Subjects also had swollen and tender joint counts recorded. Erythrocyte Sedimentation Rate and C-Reactive Protein were measured.
\end{abstract}

Results: No clinical or statistically significant improvement in QuickDASH, the primary study outcome, was noted. A minor trend in improvement was noted in shirt button time, but this improvement was not statistically significant. All other outcomes did not demonstrate any clinically or statistically significant trends.

Conclusion: No clinical improvements in the various functional and clinical parameters studied were noted. The lack of effect of canakinumab and the small size of the trial should not dissuade future research.

Keywords: Erosive Osteoarthritis; Canakinumab

\section{Introduction}

Erosive hand Osteoarthritis (EOA) is a subtype of osteoarthritis (OA) which manifests as pain, swelling, redness, and warmth and is associated with radiographic changes such as central collapsing erosions and gull wing deformity. EOA eventually leads to joint deformity and ankylosis. Despite greater analgesic use, patients with EOA have worse functional outcome and more pain than those with controlled inflammatory arthritis such as Rheumatoid Arthritis or Psoriatic Arthritis [1]. Pain scores remained significantly higher in patients with erosive OA after Compared to OA of the hands, EOA "is an important cause of disability, usually compared to the handicap caused by Rheumatoid Arthritis" [2]. The proinflammatory cytokines interleukin-1 beta (IL$1 \beta$ ) and to a lesser extent tumor necrosis factor alpha (TNF- $\alpha$ ) play a key role in the destruction of the cartilage matrix in OA [3]. In the current medical literature the use of disease modifying agents such as hydroxychloroquine in patients with EOA is based on a small retrospective study of 8 patients reported in the mid-1990s [4]. No other literature exists to support use of other disease modifying anti-rheumatic drugs (DMARDs) such as methotrexate or leflunomide presumably due to the higher risk of toxicity with these agents. A small trial of 14 patients treated with the peroxisome proliferator-activated receptor alpha agonist fenofibrate experienced significant decreases in pain score, tender joint count, duration of morning stiffness, disease activity score, Cochin index, and Erythrocyte Sedimentation Rate (ESR) [5]. An open label trial of adalimumab administered every other week treating 12 patients for 12 weeks demonstrated only a statistically significant improvement in swollen joint counts but not in other outcome measures such as pain or disability [6]. A small (3 patient) case series retrospectively reporting improvement of EOA symptoms with use of anakinra (Kineret ${ }^{\oplus}$ ) reported encouraging results after 3 months of treatment [2].

Canakinumab is a recombinant, human anti-human-IL-1 $\beta$ monoclonal antibody that belongs to the IgG1/ $\kappa$ isotype subclass [7]. It has been approved for treatment of the Cryopyrin Associated Periodic Syndromes (CAPS). Cryopyrin regulates the protease caspase- 1 and controls the activation of interleukin- 1 beta (IL-1 $\beta$ ). Canakinumab binds to human IL- $1 \beta$ and neutralizes its activity by blocking its interaction with IL-1 receptors, but it does not bind IL- $1 \alpha$ or IL-1 receptor antagonist (IL-1Ra). Since cartilage inflammation in OA is driven by IL-1 $\beta$, it was hypothesized that canakinumab (Ilaris ${ }^{\oplus}$ ) would target this cytokine in patients with EOA, improving pain and restoring function. 


\section{Methods}

This study was a 12 week prospective trial with the intent of demonstrating that canakinumab (Ilaris ${ }^{\circledast}$ ) was an effective and safe treatment for symptoms and functional decrements related to EOA. It should be noted that a true treatment effect should include structure modification. The study duration of 12 weeks was chosen as it was anticipated that some of the therapeutic efficacy would be evident at the week 12 evaluation when symptoms worsened (as canakinumab has a dosing schedule of every 8 weeks). Subjects were recruited between July 2011 and June 2014. All patients were over 18 years of age and had radiographic evidence of EOA on X-Rays of the Hands based on the criteria reported by Anandarajah [8]. To be classified as having EOA, subjects had to have OA of the Hands based on criteria established by the American College of Rheumatology [9] along with the presence of erosions in at least 2 DIP joints on plain radiographs, had a negative anti-cyclic citrullinated peptide antibody, no personal or family history of psoriatic arthritis, and no personal history of a crystalline arthropathy (serum uric acid level was normal). Subjects were excluded if they had a co-existing or different type of inflammatory arthritis (such as Rheumatoid Arthritis or Psoriatic Arthritis) or crystalline arthritis (such as polyarticular gout), moderate to severe cytopenias, were pregnant or capable of becoming pregnant, or were actively receiving therapy for neoplasia.

At their first study visit, all patients underwent a standard extensive history and physical examination. After all of the baseline study tests were performed and written consent signed, the subject received canakinumab 160 mg subcutaneously once. This dose was chosen as it is the current Food \& Drug Administration approved dose for use in the United States. Standard therapies for the treatment of OA and EOA, such as acetaminophen and/or non-steroidal anti-inflammatory drugs, were not withheld. For study visits that occurred at week 4, week 8, and week 12 (final visit), a more focused history and physical examination was performed

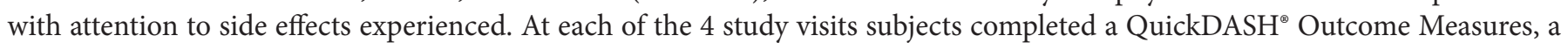
Health Assessment Questionnaire-Disability Index (HAQ-DI) with Visual Analogue Scale pain score (0 - 100), performed 3 grip strength assessments using a dynamometer (with average calculated), and performed a shirt button time. Subjects also had swollen (SJC) and tender joint count (TJC) recorded and an Erythrocyte Sedimentation Rate (ESR) and C-Reactive Protein (CRP) were obtained at each study visit. X-Rays of the Hands were obtained at the first visit only if they had not been obtained within 3 months of entry into the study.

The primary study objective was to demonstrate improvement in QuickDASH ${ }^{\otimes}$ scores at week 8. While this is not a validated outcome measure of hand OA, the QuickDASH ${ }^{\odot}$ was used as changes in the hand can affect the mechanics of the entire upper extremity. The QuickDASH ${ }^{\oplus}$ is also a valid, reliable and responsive measure which can be used for clinical and/or research purposes and some items ask about fine-motor hand functions [10]. The minimal clinically important difference for the QuickDASH ${ }^{\bullet}$ is 8.0 [10]. Secondary study objectives were to (1) demonstrate global improvement in the patient's arthritic symptoms as measured using the Health Assessment Questionnaire-Disability Index (HAQ-DI) and Visual Analogue Scale (VAS) for pain, (2) improvement in grip strength, (3) improvement in shirt button time, (4) improvements in ESR/CRP, and (5) improvement in SJC and TJC by week 8. Pre and post treatment measured variables such as QuickDASH ${ }^{\oplus}, \mathrm{HAQ-DI}$ and VAS pain scores, grip strength, shirt button test, and lab results were compared with a t-test, with each subject serving as their own control. Counted variables such as SJC and TJC were compared pre and post treatment with the Mann-Whitney $\mathrm{U}$ test. Written informed consent was obtained from all the study participants and the study was approved by the institutional review board at our medical facility.

\section{Results}

Over the 3 year period of the study, 20 subjects were evaluated but only 3 expressed interest in participating in the trial. Reasons why subjects declined enrollment included concerns about immunosuppression related to receiving the study medication, side effects that could be experienced from the study medication, or lack of any known improvement in pain or function with use of the study medication. Subject 1 was male and 76 years old at the time of enrollment. Subject 2 was a female, age 76 at the time of enrollment, and Subject 3 was female and 88 years old at the time of enrollment. Regarding the primary outcome of the study, no statistically significant improvement in the QuickDASH was noted at 8 weeks (Table 1). While only a minor trend in improvement was noted in the subjects shirt button time, this improvement was not statistically significant (Table 1). All other outcomes such as the HAQ-DI, pain score, grip strength, SJC, TJC, ESR, and CRP did not demonstrate any clinically or statistically significant improvements (HAQ-DI, pain score, and grip strength averages shown in Table 1). No adverse events were reported by any of the three subjects at any time.

\begin{tabular}{|c|c|c|c|c|}
\hline \multicolumn{5}{|c|}{ PRIMARY OUTCOME } \\
\hline QuickDASH & Week 0 & Week 4 & Week 8 & Week 12 \\
\hline Subject 1 & 6.8 & 7.5 & 12.5 & 15.9 \\
\hline Subject 2 & 59.1 & 52.3 & 45.0 & 43.2 \\
\hline Subject 3 & 25 & 0 & 0 & 2.3 \\
\hline
\end{tabular}




\begin{tabular}{|c|c|c|c|c|}
\hline \multicolumn{5}{|c|}{ SECONDARY OUTCOMES } \\
\hline Shirt Button Time & & & & \\
\hline Subject 1 & $37.5 \mathrm{sec}$ & $28.6 \mathrm{sec}$ & $19.6 \mathrm{sec}$ & $27.1 \mathrm{sec}$ \\
\hline Subject 2 & $22.3 \mathrm{sec}$ & $18.5 \mathrm{sec}$ & $20.5 \mathrm{sec}$ & $34.1 \mathrm{sec}$ \\
\hline Subject 3 & $36.5 \mathrm{sec}$ & $28.9 \mathrm{sec}$ & $35.9 \mathrm{sec}$ & $27.9 \mathrm{sec}$ \\
\hline $\begin{array}{c}\text { Average Grip } \\
\text { Strength }\end{array}$ & & & & \\
\hline Subject 1 & $53.3 \mathrm{lbs}$ & $48.6 \mathrm{lbs}$ & $58.5 \mathrm{lbs}$ & $54.9 \mathrm{lbs}$ \\
\hline Subject 2 & $22.5 \mathrm{lbs}$ & $23.7 \mathrm{lbs}$ & $21.9 \mathrm{lbs}$ & $26.0 \mathrm{lbs}$ \\
\hline Subject 3 & $33.1 \mathrm{lbs}$ & $29.2 \mathrm{lbs}$ & $35.6 \mathrm{lbs}$ & $34.2 \mathrm{lbs}$ \\
\hline HAQ-DI & & & & 1.0 \\
\hline Subject 1 & 0.75 & 0.5 & 1.0 & 1.88 \\
\hline Subject 2 & 1.88 & 1.88 & 2.0 & 0.125 \\
\hline Subject 3 & 0 & 0 & 0 & \\
\hline $\begin{array}{c}\text { Pain Score (scale 0 } \\
\text { to 100) }\end{array}$ & & & & 0 \\
\hline Subject 1 & 6 & 1 & 24 & 10 \\
\hline Subject 2 & 60 & 50 & 80 & 0 \\
\hline Subject 3 & 95 & 10 & & 0 \\
\hline
\end{tabular}

Key: Subject 1 was male, 76 years old at the time of enrollment; Subject 2 was female, age 76 at the time of enrollment; Subject 3 was female, 88 years old at the time of enrollment; Sec = seconds; HAQ-DI = Health Assessment Questionnaire-Disability Index

Table 1: QuickDASH Primary and Secondary outcome of the studies

\section{Discussion}

Given the small size of this trial it is difficult to draw any conclusions about the efficacy or lack thereof regarding canakinumab in the treatment of symptoms and functional decrements related to EOA. As stated earlier, it should be noted that a true treatment effect should include structure modification. It is possible that the lack of effectiveness of canakinumab in the treatment of EOA in this study was due to the longevity of the symptoms and functional limitations that subjects enrolled in this trial experienced. All 3 subjects had hand OA symptoms for at least 5 years, with two of the three having symptoms for 10 years or more. Perhaps the subjects had "refractory" disease and biologic therapy would not be effective, whereas biologic therapy earlier in the course of EOA would be more effective. It is also possible that ongoing use of analgesics by the subjects in this trial masked any treatment effect. Future trials will have to determine not only effectiveness of therapy but when is the optimal window to start therapy (early vs longstanding disease). Other limitations of this study are that there was no placebo control or blinding undertaken.

This study was undertaken because of the author's desire to identify a medication for this debilitating form of hand arthritis as there are currently no meaningful pharmacologic therapies for EOA. With hand OA estimated to affect $70 \%$ of adults over age 65 , the prevalence of EOA is $5-15 \%$ of those with symptomatic hand OA [8]. In the author's practice, there are enough patients with EOA but seemingly little that can be done, a frustrating situation to be in when great strides have been made in other areas of rheumatology. Routine clinical practice favors hydroxychloroquine because of the favorable risk/benefit profile of the medication and the experience reported by Bryant et al [4]. In their 2012 recommendations for the use of pharmacologic therapies for hand OA, the American College of Rheumatology did not recommend for or against use of hydroxychloroquine in EOA, citing the absence of evidence from randomized clinical trials to support a benefit of use [11]. It is author's experience that hydroxychloroquine does little to control the pain or functional limitation of EOA. This study was also undertaken because the pathophysiologic role of IL$1 \beta$ in OA of the hand supports a role for IL- $1 \beta$ blockade. The success that Bacconnier et al. experienced with anakinra suggested that targeting IL-1 $\beta$ with canakinumab could be successful. Indeed, two clinical trials have just been completed utilizing the medication gevokizumab, a more potent IL-1 $\beta$ inhibitor, in the treatment of EOA [12]. These phase 2, proof-of-concept studies have collectively enrolled 178 patients who were randomized and blinded to either the study medication or placebo. Even if the outcome of these trials does not favor use of gevokizumab in EOA, these two trials will still be trailblazing as they will represent the best designed and largest trials performed to date for this seemingly forgotten form of debilitating arthritis.

\section{References}

1. Wittoek R, Cruyssen BV, Verbruggen G (2012) Predictors of functional impairment and pain in erosive osteoarthritis of the interphalangeal joints: comparison with controlled inflammatory arthritis. Arthritis Rheum 64: 1430-6.

2. Bacconnier L, Jorgensen C, Fabre S (2009) Erosive osteoarthritis of the hand: clinical experience with anakinra. Ann Rheum Dis 68: 1078-9.

3. Kobayashi M, Squires GR, Mousa A, Tanzer M, Zukor DJ, et al. (2005) Role of interleukin-1 and tumor necrosis factor alpha in matrix degradation of human osteoarthritic cartilage. Arthritis Rheum 52: 128-35. 
4. Bryant LR, Des Rosier KF, Carpenter MT (1995) Hydroxychloroquine in the treatment of erosive osteoarthritis. J Rheumatol 22: $1527-31$.

5. Shirinsky IV, Shirinsky VS (2014) Treatment of erosive osteoarthritis with peroxisome proliferator-activated receptor alpha agonist fenofibrate: a pilot study. Rheumatol Int 34: 613-6.

6. Magnano MD, Chakravarty EF, Broudy C, Chung L, Kelman A, et al. (2007) A pilot study of tumor necrosis factor inhibition in erosive/inflammatory osteoarthritis of the hands. J Rheumatol 34: 1323-7.

7. Kuemmerle-Deschner JB, Haug I (2013) Canakinumab in patients with cryopyrin-associated periodic syndrome: an update for clinicians Ther Adv Musculoskelet Dis 5: 315-29.

8. Anandarajah A (2010) Erosive osteoarthritis. Discov Med 9: 468-77.

9. Altman R, Alarcón G, Appelrouth D, Bloch D, Borenstein D, et al. (1990) The American College of Rheumatology criteria for the classification and reporting of osteoarthritis of the hand. Arthritis Rheum 33: 1601-10.

10. Angst F, Schwyzer HK, Aeschlimann A, Simmen BR, Goldhahn J (2011) Measures of adult shoulder function: Disabilities of the Arm, Shoulder, and Hand Questionnaire (DASH) and its short version (QuickDASH), Shoulder Pain and Disability Index (SPADI), American Shoulder and Elbow Surgeons (ASES) Society standardized shoulder assessment form, Constant (Murley) Score (CS), Simple Shoulder Test (SST), Oxford Shoulder Score (OSS), Shoulder Disability Questionnaire (SDQ), and Western Ontario Shoulder Instability Index (WOSI). Arthritis Care Res 63: S174-88.

11. Hochberg MC, Altman RD, April KT, Benkhalti M, Guyatt G, et al. (2012) American College of Rheumatology 2012 recommendations for the use of nonpharmacologic and pharmacologic therapies in osteoarthritis of the hand, hip, and knee. Arthritis Care Res 64: 465-74.

12. U.S. National Institutes of Health (2012) Safety and Biologic Activity Study of Gevokizumab to Treat Erosive Osteoarthritis of the Hand, USA.

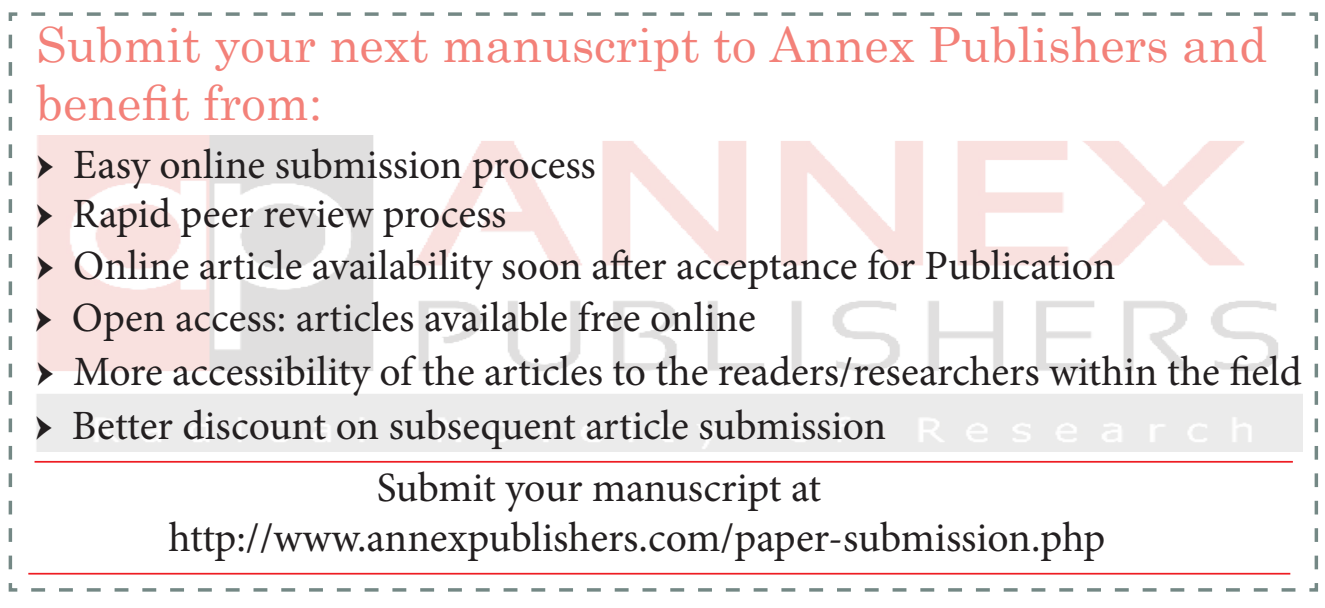

